\title{
Interventions to Decrease Inappropriate Antibiotic Use for Non-acute Respiratory Illness in Long-Term Care Settings
}

\author{
Joy M. Barnes ${ }^{1} \&$ Pamela Bradshaw ${ }^{2}$ \\ ${ }^{1}$ Bethel University School of Nursing, Mishawaka, Indiana, USA \\ ${ }^{2}$ Shannon-Medical Center, San Angelo, Texas, USA \\ Correspondence: Samuel P. Abraham, Associate Professor of Nursing, Bethel University School of Nursing, \\ 1001 Bethel Circle, Mishawaka, Indiana, 46545, USA.
}

Received: June 1, 2019

Accepted: June 19, 2019

Online Published: June 21, 2019

doi:10.20849/ijsn.v4i3.599

URL: https://doi.org/10.20849/ijsn.v4i3.599

\begin{abstract}
Background: The life-saving power of antibiotics could be lost forever if leaders fail to implement effective antibiotic stewardship programs at all healthcare levels. Grahams' Knowledge to Action theory guided the development of an antibiotic stewardship program in a long-term care facility that had received a citation for having no active antibiotic stewardship program as required by federal regulations. Purpose: The purpose of this project was to develop and implement an evidence-based antibiotic stewardship program into one long-term care facility. Methods and Materials: This quality improvement project was a population-based systems charter development. The implementation intervention was designed to change the way health care professionals treat non-acute episodes of upper respiratory infections in a long-term care setting. This project utilized the suspected lower respiratory infection (LRI) Situation, Background, Assessment, Recommendation (SBAR) form to reduce the number of antibiotics given during the early part of cold and influenza season of 2018. An antibiotic stewardship policy was developed by multidisciplinary team members and then implemented into the facilities daily practice. Results: The point-prevalence rate of antibiotics within this facility dropped from $24 \%$ in 2017 to 6\% in 2018 after implementation of the antibiotic stewardship program. Conclusion: This project demonstrates how long-term care facilities can successfully implement an antibiotic stewardship program and potentially improve overall healthcare outcomes for the residents.
\end{abstract}

Keywords: antibiotic stewardship, long-term care, lower respiratory infections, influenza

\section{Introduction}

Antibiotic-resistant microbes will remain a health concern for all, unless intentional plans are in place to change current prescribing practices in nursing facilities across the nation (Ashford and Cook, 2016; Voelker, 2015). The current antibiotic prescribing practices (CDC, 2017; Fleming et al., 2015) and the unnecessary use of antibiotic treatment in long-term care facilities is contributing to the increase in global antibiotic-resistant microbes (CDC, 2017; Fleming et al., 2015). Research has demonstrated that antibiotic use, misuse, and overuse in the long-term care industry can negatively affect healthcare outcomes for residents within the facilities (Ashraf \& Cook, 2016; CDC, 2017; Crnich, et al., 2015; Dar et al., 2016).

The overall aim of this project was to measure a reduction in the number of inappropriate antibiotics prescribed for non-acute respiratory infections during the first half of the cold and influenza season in the fall of 2018 in one long-term care facility in Indiana.

\subsection{Problem Description}

In a 62-bed nursing home in Indiana, there was an urgent need to develop an antibiotic stewardship program. During the annual state survey process on January 9, 2018, the facility received a citation for failing to demonstrate the appropriateness and effectiveness of antibiotics for residents throughout the year (Administrator, personal communication, January 15, 2018). Two specific deficiencies were noted for this facility during the survey process. First, the facility did not have policies and procedures that establish minimum criteria for initiating antibiotics (Administrator, personal communication, January 15, 2018). Second, the current policies and procedures were not consistent with current evidence-based standards of practice for antibiotic use (Administrator, personal communication, January 15, 2018). 
Federal regulation 483.80 demanded that nursing facilities have an antibiotic stewardship program in place by the end of 2017 (Office of Federal Register, 2016). The facility had no antibiotic stewardship program in place (Administrator, personal communication, January 15, 2018) and development of an antibiotic stewardship program was a high priority due to the current non-compliance of the facility with a federal requirement (Administrator, personal communication, January 15, 2018).

Specific data related to antibiotic use in this facility during the last quarter of 2017 further support the gap in practice in this long-term care setting. A review of infection control reports obtained by the pharmacist and the director of nursing revealed a high use of antibiotics in this facility. In October $2017,60 \%$ of residents received a ten to fourteen-day treatment of antibiotic for infections (Director of Nursing, personal communication, January 15 , 2018). Specifically, five residents were treated for upper respiratory infections with no supporting documentation to indicate a need for antibiotic use.

In November 2017, antibiotic use increased to $74 \%$ of the residents receiving a ten-day antibiotic treatment for a variety of infections. There were 160 days of antibiotic treatment for the facility in the month of November. This number reveals that sixteen of the sixty-two residents had a ten-day antibiotic treatment for a known or suspected infection (Director of Nursing, personal communication, January 15, 2018). Eight residents were treated for respiratory infections with no supporting documentation to indicate a need for antibiotic use (Director of Nursing, personal communication, January 15, 2018).

An exploration of the December 2017 infection report revealed that $54 \%$ of residents received antibiotic treatment (Director of Nursing, personal communication, January 15, 2018). Four residents were treated for respiratory infections during December 2017, however only two of the residents had supporting documentation to indicate a need for antibiotic use (Director of Nursing, personal communication January 15, 2017).

\section{Support From Literature}

The Center for Disease Control and Prevention (CDC) reports that $70 \%$ of nursing home residents are prescribed at least one antibiotic every year (CDC, 2017). Seventy-five percent of the residents that received antibiotics did not benefit from antibiotic treatment because the antibiotics were not necessary for treatment of the illness the resident had developed (CDC, 2017; Fleming et al., 2017).

Implementation of a formal antibiotic stewardship program in long-term care facilities has demonstrated to decrease the number of unnecessary antibiotics prescribed for the residents (Crnich et al., 2015; Dar et al., 2016; Squires et al., 2014; Voelker, 2016). However, long-term care facilities across the nation have been slow to adapt and embrace this new evidence-based practice to decrease inappropriate use of antibiotics (Crnich et al., 2015; Sloane, Huslage, Kistler, \& Zimmermann, 2016; Voelker, 2016).

The Centers for Medicare and Medicaid Services (CMS) has recognized that many nursing homes need to do more to prevent and appropriately treat infections for residents living in long-term care facilities (CDC, 2017). In fact, new regulations went into effect January 1, 2018 to address the problem of inappropriate use of unnecessary antibiotics (CMS, 2017; Crnich, et al., 2015). The new regulations will drive antimicrobial stewardship principles into the long-term care setting (CDC, 2017; CMS, 2016; Crnich et al., 2015; Dar et al., 2016). As part of the response to the CMS mandate to improve appropriate antibiotic use within the long-term care industry (CDC, 2017; CMS, 2016), this project developed an antibiotic stewardship program in a local long-term care facility.

Research identifies several current prescribing problems that result in unwarranted use of antibiotics for residents in long-term care centers. First, it has been shown that several residents receive antibiotics that are not susceptible to treatment of the microbe identified to cause the infection (CDC, 2017; Crnich et al., 2015; Dar et al., 2016). Empiric therapy is often used as the first treatment choice for the long-term care resident (Dar et al., 2016; Fleming et al., 2015). Next, studies found that antibiotic treatment was unnecessary due to the viral nature of the illness (CDC, 2017; Fleming et al., 2015). Finally, research showed residents in long-term care facilities consistently received antibiotics for more days than research-based guidelines recommended for treatment (Fleming et al., 2015). It is a widespread practice for long-term care residents to take antibiotics for ten to fourteen days at a time (CDC, 2017; Fleming, et al., 2015).

Decreasing the use of unnecessary antibiotics within this facility will ultimately decrease the risk of the development of antibiotic resistance within this population and will improve overall healthcare outcomes for the residents (CDC, 2017; CMS, 2016; Crnich, et al., 2015; Dar et al., 2016).

\subsection{Available Knowledge}

Keywords used in the initial search of the literature were antibiotic stewardship, long-term care, overuse of 
antibiotics, misuse of antibiotics, upper respiratory infections and policy. Further searches included the following keywords: upper respiratory infection treatment, healthcare regulations and antibiotic stewardship, family perception of antibiotic use, fiscal impact and antibiotic stewardship programs, and adverse side effects and antibiotics.

Research was limited to publications from 2014 - 2018 in the English language. The electronic databases of PubMed, OVID, and CINHAL were used to search for peer-reviewed articles. This search yielded sixty-one articles to review. Furthermore, a revision of the keyword "long-term care" was changed to "nursing homes". This change in the keyword assisted this researcher to identify fifteen additional articles related to successful development of an antibiotic stewardship program within the long-term care environment.

Next, articles were excluded when the study focused on long-term acute care settings within a hospital, if the article specifically addressed other infection types (i.e. UTI), and if the article could not specifically provide information (positive or negative) about the overall impact of the antibiotic stewardship program. Using the above process in researching the PICOT question, a variety of articles were considered for this project including integrative reviews, descriptive (correlational) studies, a longitudinal analysis, an instrumental variables approach, and several pilot projects based on clinical guidelines. The total number of articles included in the review of literature is twenty-three.

\subsection{Collaboration Among Disciplines}

The long-term care team is made up of several different disciplines. An essential component of creating change in the long-term care setting includes the understanding that several disciplines must be involved in the development phase of any new policy or program for antibiotic stewardship (Crnich et al., 2015; Squires et al., 2014). Goal setting and a shared vision will create a solid foundation for a sustainable antibiotic stewardship program and truly change the utilization of antibiotics in the long-term care setting (Dar et al., 2016, Voelker, 2016).

\subsection{Resident and Family}

Resident and family participation is essential when changing the current practice of antibiotic use within the facility (Crnich, et al., 2015; Squires et al., 2014). Extensive application of the Core Elements of Antibiotic Stewardship Program flyers and resident/family education tools, developed by the CDC (2018), were utilized to engage residents and family members in understanding the dangers of misuse or overuse of antibiotics for non-acute respiratory illness. This was a crucial step in creating a culture, within the facility, to encourage appropriate use of antibiotics (Crnich et al., 2015).

Residents and family members were an active part of the project charter. Identification and recruitment of residents and family members began by attending a resident council meeting to bring awareness about the need for change within the facility regarding antibiotics. To encourage participation in the project charter committee, follow-up communication with the resident and the family members via written and verbal interaction occurred.

\subsection{Suspected (Lower Respiratory Infection) LRI SBAR Tool}

Communication between the nurse and the prescribing provider has been observed to be a leading factor in misuse of antibiotics in the long-term care setting (AHRQ, 2017, Dar et al., 2016, Squires et al., 2014). Often in facilities across the nation, the provider prescribes antibiotics based on very few criteria gathered by the nurse (Crnich et al., 2015). Thus, the suspected LRI SBAR tool was developed by the AHRQ in 2017 to establish basic guidelines to initiate antibiotics when residents exhibit respiratory symptoms (ARHQ, 2017). The LRI SBAR tool has been proven to be effective in improving communication between the nurse and the prescriber (Crnich et al., 2015; Dar et al., 2016) and encourages the nurse and prescriber to collaboratively work together to identify specific factors that would indicate a need for or against antibiotic treatment (ARQH, 2017, Squires et al., 2014).

\subsection{Impact on Patients/Families}

A chief contributing factor to antibiotic resistance with the long-term care setting has been the overuse, misuse, or inappropriate use of antibiotics (Ashraf \& Cook 2016; CDC, 2017; Crnich et al., 2015; Dar et al., 2016). More than half of nursing home residents are prescribed at least one systemic antibiotic to treat an illness every year (CDC, 2017; Fleming et al., 2015). The CDC found that of the antibiotics prescribed to residents in long-term care facilities, $75 \%$ were not appropriate to treat the illness because the prescribed antibiotic was not susceptible to the present bacteria, minimal clinical data was collected to determine need for antibiotic treatment, or it was viral in nature (CDC, 2017; Crnich et al., 2015; Pogue, 2015).

To prevent further negative healthcare outcomes in the long-term care setting, it is imperative to appreciate the 
complications of misuse, overuse, and inappropriate use of antibiotics (Ashraf \& Cook, 2016; CDC, 2017; Crnich et al., 2015; Dar et al., 2016). Understanding that dire consequences can occur when antibiotics are inappropriately utilized in the long-term care setting is an essential component when initiating a change in current prescribing practices (CDC, 2017; Doron, Nadkami, Lyn, Lawarance, Dvidson, Evans \& Snydman, 2013; Fleming et al., 2015). Three main problems will be examined for this literature review; antibiotic resistance, development of other potentially fatal infections, and the overall increase in the cost of healthcare.

\subsection{Increase in Antibiotic Resistance}

Antibiotic resistance is reported to be a grave global health concern (Ashraf \& Cook, 2016; CDC, 2017; Doron et al., 2013; Dar et al., 2016). In the United States alone, the CDC (2017) reports that antibiotic resistance accounts for approximately two million infections and 23,000 deaths per year (Lim, Stuart, \& Kong, 2015; Sanchez, Fleming-Dutra, Roberts, \& Hicks, 2016). Research reveals that antibiotic resistance is increasing in the long-term care environment across the United States (Hertz, 2013; Fleming et al., 2015; Lenhoff, 2016).

Antibiotic resistance has been described as just enough change in the makeup of the bacteria to fight off the effects of the antibiotics that previously debilitated this specific bacterium (Lenhoff, 2016; Pogue, 2015). This change allows the bacteria to thrive in the body and renders the antibiotic useless (Ashraf \& Cook, 2016; CDC, 2017; Crnich et al., 2015; Dar et al., 2016).

This alteration provides an opportunity for the newly mutated microbe to cause havoc within the body (Ashraf \& Cook, 2016; CDC, 2017; Doron et al., 2013). One major consequence of antibiotic resistance is that there may soon be a time when no treatment is available to improve the patient healthcare outcomes (Ashraf \& Cook, 2016; CDC, 2017). Everyday procedures, such as surgery, may have dismal consequences if we lose the power of antibiotics in destroying common bacterial infections (Ayree, 2015; Doron et al., 2013).

\subsection{Development of Potentially Fatal Infections}

When several antibiotics are prescribed to treat an infection, development of side effects may cause complications to the patient (Charani \& Holmes, 2013; Daneman et al., 2017 Hertz, 2015). It is well documented that antibiotics disrupt the microbes in the gastrointestinal tract and can cause a serious infection known as clostridium difficile (Charani \& Holmes, 2013; Daneman et al., 2017; Dubberke et al., 2016).

In 2011 there were 453,000 healthcare-acquired clostridium difficile infections (Daneman et al., 2017). Treatment for clostridium difficile, which frequently included inpatient hospitalization, is reported to be at least $\$ 4.8$ billion dollars (Daneman et al., 2017; Dubberke et al., 2016). Even with treatment for clostridium difficile infection, in 2011, over 29,000 people were unable to recover from this infection and died (Charani \& Holmes, 2013; Daneman et al., 2017).

Deaths from clostridium difficile are expected to decrease when antibiotic stewardship programs are effectively placed in clinical practice (Daneman et al., 2017; Dubberke et al., 2016). Long-term care facilities must play an active role in development and implementation of antibiotic stewardship programs to decrease the potential of development of clostridium difficile (Charani \& Holmes, 2013; Daneman et al., 2017; Hertz, 2015).

\subsection{Increase in Healthcare Costs}

Complications of overuse of antibiotics can result in additional health care expense for the patient (Diamond, 2016; Dyar, 2015; Gandra, 2014). For example, inpatient hospitalization and treatment for clostridium difficile require expensive antibiotics and fluids to treat dehydration and the infection (Ashford \& Cook, 2016; Dyar, 2015). When long-term care healthcare prescribers utilize evidence-based guidelines when prescribing antibiotics, health care costs will decrease (CDC, 2017; Doron et al., 2013; Fleming et al., 2015).

\subsection{Impact on Quality}

Migration to continuum care communities is presenting a new challenge for care providers to prevent infection within the communities (Neville et al., 2016; Hertz, 2015.) To ensure high-quality care, a focus on appropriate use of antibiotics will prevent negative healthcare outcomes in the new healthcare communities (Neville et al., 2016; Hertz, 2015; Van de Berg et al., 2015). Patients, families, and healthcare providers must work together to understand the importance of appropriate use of antibiotics within the community to ensure a high-quality, long-life for the residents (Lim, Stuart, \& Kong, 2015; Sanchez, Fleming-Dutra, Roberts, \& Hicks, 2016).

Implementation of a formal antibiotic stewardship program in long-term care facilities has demonstrated a decrease in the number of unnecessary antibiotics prescribed for the residents (Crnich et al., 2015; Dar et al., 2016; Squires et al., 2014; Voelker, 2016). The time is now to demonstrate that antibiotic stewardship programs in long-term care facilities will improve the overall healthcare of the residents of long-term care communities 
across the nation (Dar et al., 2016; Squires et al., 2014; Voelker, 2016).

\subsection{Healthcare Policy}

Olans, Nicholas, Hanley and DeMaria, (2015) found that engagement of the residents and the family members is vital to the success of an antibiotic stewardship program within long-term care facilities. In 2015, antibiotic stewardship gained momentum in the United States. The Obama Administration orchestrated a White House event to bring awareness and begin a discussion of the need for antibiotic stewardship across all healthcare service-lines, including long-term care facilities (Charani \& Holmes, 2013; Olans et al., 2015). This assembly proved to be productive in addressing antibiotic stewardship awareness in this country. Action plans to educate the public about the global concerns of antibiotic resistance were funded and developed (Charani \& Holmes, 2013; Olans et al., 2015).

The CDC, World Health Organization (WHO) and The Joint Commission partnered to launch an awareness campaign in November of 2016. The "Get Smart: Know When Antibiotics Work" Program began a campaign to inform the public about the current prescribing practices of antibiotics (CDC, 2017). This program laid the foundation for resident and family involvement in understanding when antibiotics should be utilized and when it can be dangerous (CDC, 2017).

\subsection{Summary of Literature Review}

A fundamental change to the current prescribing practices of antibiotics in the long-term care setting is an essential step to reduce antibiotic resistance and prevent negative healthcare outcomes worldwide (Crnich et al., 2015; Dar et al., 2016; Squires et al., 2014). The precious resource of powerful antibiotics to treat common infections could be lost forever if healthcare providers continue to prescribe antibiotics that are unnecessary (Squires et al., 2014).

Action must take place immediately to impact the future of healthcare and prevent complications from the misuse of antibiotics (Crnich et al., 2015; Dar et al. 2016). A change in current practice has the potential to save lives and to decrease healthcare costs of medical complications related to inappropriate use of antibiotics (CDC, 2017; Doron et al., 2013; Fleming et al., 2015).

If nursing homes fail to change the current practice of prescribing unnecessary antibiotics, the likelihood of decreasing global antimicrobial resistance is minimal (Voelker, 2015). Long-term care residents, as well as all citizens of the globe, remain at risk for negative healthcare outcomes related to unnecessary use of antibiotics (Crnich et al., 2015; Dar et al., 2016, Squires et al., 2014) if current prescribing trends remain unchanged.

\section{Rationale}

The Knowledge to Action Framework (KTA) (Graham et al., 2006) was used to guide the development and implementation of an antibiotic stewardship program in the long-term care facility. The KTA describes two processes that work together to improve the way evidence-based research knowledge is integrated into practice (Graham et al., 2006). One process focuses on knowledge creation (Graham et al., 2006) and the other process focuses on action. Together, the two processes will guide additional information to be adopted by the culture, sustain the evidence-based practice and potentially change the face of health care (Graham et al., 2006).

The KTA framework was a useful guide in the development of this research project. The always fluid and dynamic KTA framework was chosen due to the complexity of the nursing home environment and the desperate need to make changes in this specific healthcare system (Graham et al., 2006). The KTA framework has proven to be effective as a quality improvement program for long-term care facilities (Cranley, Norton, Cummins, Barnard \& Eastbrook, 2012; Squires et al., 2016; Voelker, 2015).

This robust framework begins with gathering knowledge on current evidence-based practice to improve patient care. The project manager explored evidence available in the literature and practice guidelines to drive the development and creation of an antibiotic stewardship program in a long-term care setting. Next, the KTA framework provides an opportunity to develop a tailored intervention for the specific long-term care environment based upon the unique culture within the facility (Graham et al., 2006). The facility-specific intervention can be funneled into a customized antibiotic stewardship program that can be initiated in the long-term care environment. Finally, the KTA framework provides an on-going evaluation phase to ensure the new knowledge is adapted into the current highly regulated environment of long-term care (Graham et al., 2006).

\subsection{Study Variables}

$>$ Will residents and family members refuse to accept the new guidelines?

$>$ Will staffing challenges impact the communication between the nurse and the prescribing providers? 
$>$ Will nursing staff embrace the suspected LRI SBAR form?

$>$ Will the facility have an opportunity to utilize the new antibiotic stewardship program soon after roll-out of new program?

\subsection{Study Assumptions}

$>$ All facility staff, residents, family members and prescribing providers are willing to participate in efforts to decrease unnecessary antibiotic treatment for residents within a long-term care facility.

$>$ The nurses and prescribing providers will work together and communicate to ensure that the practice change is integrated into the everyday practice at this facility.

$>$ The facility administration will support the practice change to decrease the use of unnecessary antibiotics and improve overall healthcare outcomes for residents of the facility.

\subsection{Specific Aims}

The overall aim of this project is to measure a reduction in the number of inappropriate antibiotics prescribed for non-acute respiratory infections during the first half of the influenza season of fall 2018 in one long-term care facility in Indiana.

\section{Methods add Materials}

\subsection{Context}

This project encouraged a cultural shift in the thinking of the interdisciplinary team members, administration, and the facility staff to meet regulatory requirements and to sustain the practice of antibiotic stewardship within the facility (Dar et al., 2016, Olans et al., 2015; Squires et al., 2014). Furthermore, successful implementation and adaptation of the antibiotic stewardship program in this facility influenced the facilities around the community. This facility has set the standard by becoming a model of care for long-term care and demonstrating an effective cultural shift within the facility and industry concerning antibiotic prescribing practices (Charani \& Holmes, 2013; Olans et al., 2015).

\subsection{Interventions}

The project design for this evidence-based practice project was the development of a project charter and subsequent implementation of an antibiotic stewardship program for residents, families, clinical care providers and prescribers in a long-term care facility. This project charter development and implementation was based on research data demonstrating that utilization of evidence-based suspected LRI SBAR targeted form, multidisciplinary team development of policy, and inclusion of resident, family and clinical staff education will decrease the use of unnecessary antibiotics in the long-term care setting (Crnich et al., 2015; Dar et al., 2016, Squires et al., 2014).

A review of historical antibiotic prescription data from the long-term care setting was compared to post-intervention antibiotic prescription data. Furthermore, a decrease in unnecessary antibiotic use in the long-term care setting will impact the global problem of antibiotic resistance and ultimately impact healthcare outcomes worldwide (Lim, Stuart, \& Kong, 2015; Sanchez et al., 2016).

This evidence-based practice project focused on policy development and implementation of evidence-based practices into a long-term care environment. Capella University's IRB and the facilities human subject review process was completed prior to implementation of this project. No data that would identify residents was included in this project. Therefore, no human subjects needed to be protected in the collection of the data for this project.

\subsection{Sustainability of the Antibiotic Stewardship Program}

The project design was intentional to ensure ownership of the antibiotic stewardship program remains within the facility's own multidisciplinary team. The antibiotic stewardship program took root and grew within the facility and led to a cultural change. This project was built with the end in mind, knowing that the facility must engage at the start to ensure an easy transition to continued growth and sustainability of the program to impact resident healthcare outcomes for years to come.

\subsection{Project Charter - Policy Development}

Creation of the antibiotic stewardship committee was the first step in the policy development process (Minnesota Department of Health, 2001). During this phase of the project, a committee was formed to develop the antibiotic stewardship goals for the facility. Committee members included diverse healthcare providers (medical director, 
pharmacist, director of nursing, unit manager, staff nurse, certified nurse assistant, DNP learner), resident representative, family member representative, and the administrator of the facility.

Health care providers were recruited based on job or role within the facility. Identification of residents and family members interested in participating in this committee occurred during a resident council meeting. Finally, the recruitment of a staff nurse and certified nurse assistant was identified through conversations with the Director of Nursing.

Recruitment of residents and family members began by attending a resident council meeting to bring awareness to the group about the need for change within the facility regarding antibiotics. Identification of residents and family representatives to be included on the antibiotic stewardship committee began at the resident council meeting.

\subsection{Project Charter - Provider Education}

The tool selected for this project charter was the suspected LRI SBAR tool from the AHRQ (2017). The implementation phase involved educating the entire facility staff about the new antibiotic stewardship program, including the new evidence-based tool, the suspected LRI SBAR form. AHRQ granted permission for this tool to be utilized in this project improvement project (Agency for Healthcare Quality, 2018).

A communication tool for all prescribing providers informing them of the new antibiotic stewardship program was developed and mailed to all prescribers. To roll out the new antibiotic stewardship program at the facility, a face-to-face education session was presented to the nursing staff. In this education session, handouts, guides and rational to encourage positive engagement of the nursing staff was presented.

\subsection{Project Charter - Resident/Family Participation}

To encourage on-going participation in the antibiotic stewardship program, communication with the resident and the family member via written and verbal interaction occurred. Successful implementation included educating residents and families about the new practice change and the implications for the residents. Extensive application of the Core Elements of Antibiotic Stewardship Program flyers and resident/family education tools, developed by the CDC (2018), was utilized to engage residents and family members in understanding the dangers of misuse or overuse of antibiotics for non-acute respiratory illness. This was a crucial step in creating a culture, within the facility, to encourage appropriate use of antibiotics (Crnich et al., 2015). Residents and family members were an active part of the project charter implementation phase.

\subsection{Sample Inclusion Criteria}

Residents must be admitted to the participating facility in the long-term care unit, assisted living section or rehabilitation units. This allows for retrievable data of antibiotic treatment records to be reviewed and analyzed for this study. The adult day-care clients were not included for this study.

\subsection{Recruitment Process}

The only recruitment process for resident participation in this study was that the resident must be admitted to this long-term care facility and resides in the long-term care unit, assisted living section, or the rehabilitation unit.

All full time, part-time, and PRN licensed nursing staff employed at the facility, medical director, and all prescribing practitioners that care for the residents within the facility will be educated on the antibiotic stewardship program and the expectations for care will be explained at the roll-out of the program.

All residents of the facility and their family members were invited to participate in the educational program about the global problem of antibiotic resistance and the facility's antibiotic stewardship program. This was a critical step to stop the deadly increase of antibiotic-resistant microbes worldwide.

\subsection{Study of the Interventions}

Evaluation of the intervention was monitored by tracking the number of residents who experience upper respiratory symptoms, tracking the number of cases treated by antibiotics and the evaluation of the correct selection of the antibiotic prescribed. The following data was retrieved from the facility: specific number of antibiotics ordered, specific drug classification of antibiotics ordered, length of treatment days, review of documentation to see if symptoms were identified that supported the use of antibiotics, review of documentation to see if symptoms resolved, and monitor return to hospital data for residents that were prescribed and/or not prescribed antibiotics.

The information was gathered through chart review, pharmacy-dispensing records, suspected LRI SBAR record review, and infection control monitoring reports. Data was collected, from the same time frame in 2017, and 
compared to the post-intervention data. The comparison of antibiotic rates between the two years were used to determine the effectiveness of the intervention of this project.

Three approaches were used to organize and track data during this study. First, an audit of the completeness of clinical documentation at the time of antibiotic initiation was appraised. This information was utilized to determine if the clinical symptoms meet the criteria (Suspected LRI-SBAR) for an antibiotic start based on the clinical presentation at the time of evaluation of a suspected respiratory infection.

Next, an audit of the comprehensiveness of the antibiotic order was evaluated. This audit ensured that all required parts of the prescription are present at the initiation of antibiotic treatment. The audit looked for the following criteria:

$>$ Dose (including route)

$>$ Duration (start date, end date, planned days of therapy)

$>$ Indication (rational and treatment site)

Finally, an audit occurred to determine if the antibiotic that was selected is consistent with recommended antibiotics for specific infection identified. During policy development, specific guidelines for respiratory infections and treatment options were developed. This audit assisted in evaluating the consistency of prescribing practices within the facility.

\subsection{Measures}

Three measures were used to measure outcomes of the antibiotic stewardship program. First, a point-prevalence survey of antibiotic use tracked the proportion of residents receiving antibiotic therapy during the specified time frame. This project was time-limited; therefore, a point- prevalence survey was used to capture antibiotic use during the 10-week observation period. This type of evaluation captures a snap-shot of the antibiotic burden for this long-term care facility. The point-prevalence survey provides the ability to extrapolate meaningful data such as complications related to antibiotic use (i.e. C-diff,), prescriber patterns of antibiotic starts, class of antibiotics prescribed, and days of treatment.

The point prevalence survey of antibiotic use for the post-implementation of this study can easily be compared to the point-prevalence antibiotic burden for 2017 (during the same 10 -week period of 2017). Chi-Square revealed an significance in the change of practice from 2017 to 2018.

Next, antibiotic starts were calculated for the facility and assessed the impact of the antibiotic stewardship program (AHRQ, 2017; CDC, 2018). This information describes the rate of new antibiotics prescribed during the 10 -week post-implementation phase of the antibiotic stewardship program. The antibiotic rate for the 10 -week observation period was compared to the data for the same period during 2017. This was calculated by the following equation (AHQ, 2017; CDC.2018):

$>$ Rate of new antibiotic starts initiated (per 1,000 resident days) = (number of new antibiotics prescriptions/total number of resident days) X 1,000

Finally, the total antibiotic days of therapy (DOT) measurement was calculated for this study. This data can monitor changes of antibiotic use over time (AHRQ, 2017; CDC, 2018). An antibiotic day, for this study, is defined as each day a resident receives a single antibiotic. To calculate the rate of antibiotic DOT the following equation was applied (CDC, 2018):

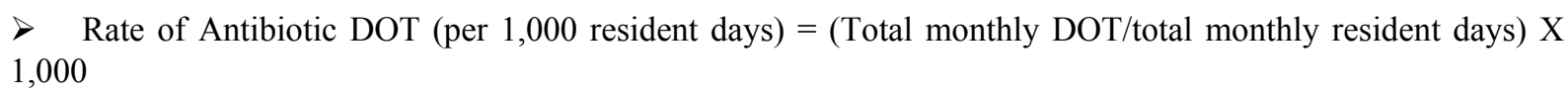

\section{Analysis}

\subsection{Limitations}

One major limitation of this project is the small sample size. This is due to the implementation site, a small, privately owned long-term care facility in the Midwest. The facility cannot expand in number and this is a known limitation with limited efforts to minimize. However, this study design has the potential to change several long-term care facilities around the nation and adaptation to larger facilities will add to the scholarly support and potential guidelines for other facilities in the future.

A second limitation of this study is to recognize that changes in policy and protocol can be met with resistance by the medical providers. This resistance occurs when practitioners/prescribers are comfortable with the existing practice that is in place within the facility. Utilization of antibiotic champions to encourage and adopt the new change will be vital and may reduce the resistance. Ensuring that this project has full administrative support will 
also be vital in increasing compliance and integration of the antibiotic stewardship program into the facility. Application of the KTA theory in adapting to a change within the facility will be of immense importance in the successful transition to an evidence-based practice guideline within this facility. Finally, a third limitation is the 10 -week time frame for this project. This short period may not be long enough to see an impact on antibiotic prescribing practices.

\subsection{Ethical Considerations}

Quality Improvement (QI) projects built on sound evidence-based practice (EBP) guidelines are essential to improve the overall quality of healthcare (Institute of Medicine, 2001; National Strategy for Quality Improvement in Healthcare, 2012). QI projects have shown to be an effective research design to promote practice change in the long-term care setting (Voelker, 2016). High-quality, ethical QI projects have the potential to advance nursing knowledge and ensure the EBP results are applied at the bedside (National Strategy for Quality Improvement in Healthcare, 2012).

\subsubsection{Physical Risks}

This study involved improving the current care for residents within the long-term care facility. A focus on improving care was accomplished by implementing the suspected SBAR-LRI tool into everyday practice at the facility. Developed by the AHRQ, minimum criterion based on scientific evidence, is used to initiate antibiotic use when respiratory symptoms are displayed by the resident (AHRQ, 2017). This study was specifically designed to stop the overuse of prescribing antibiotics for non-acute symptoms of respiratory illness. The health care providers are already actively included in making daily care decisions based on clinical data. This study did not pose a physical risk to any participant.

In fact, research demonstrates that physical harm may occur to the resident if the clinical staff prescribes an antibiotic when the standard criterion is not met (Charani \& Holmes, 2013). It is widely known that antibiotics disrupt the microbes in the gut and can cause a serious infection known as clostridium difficile (Charani \& Holmes, 2013; Daneman et al., 2017). In 2011, over twenty-nine thousand people died from this infection (Charani \& Holmes, 2013; Daneman et al., 2017).

\subsubsection{Psychological Risks}

The goal of this study was to make a fundamental change in the thinking of all the participants about appropriate antibiotic use. During this change in practice, there was minimal risk for the participants to experience increased stress levels when a clinical decision is made to not initiate antibiotics (Sloane, Huslage, Kistler, \& Zimmermann, 2016; Voelker, 2016). It is important to recognize that many participants were accustomed to receiving an order for antibiotics at any sign of respiratory illness (Voelker, 2016).

However, the participants must understand that the benefit of providing high-quality care to the residents is grounded in the ANA code of ethics. ANA provision four articulates that the nurse has the authority, accountability, and the responsibility for nursing practice that promotes health and provides optimal care (ANA, 2015; Winland-Brown, Lachman, \& Swanson, 2015). In this study, optimal care included the appropriate use of antibiotics. The study design was deliberate in minimizing the potential of increased stress among the participants by purposely including all participants in an awareness campaign and education sessions about the global antibiotic resistance crisis and what changes can be made at the local level (AHRQ, 2018).

\subsubsection{Social Risks}

The awareness and education component of this research study mitigated any invasion of privacy concerns. It is important to be transparent when conducting ethical research (Hockenberry, 2014) and all potential participants were aware that a clinical change was being implemented within the facility. All participants were made aware that a quality improvement study was being performed within the facility from the onset of the project charter. There is no risk of social harm regarding the invasion of privacy to the participants as all participants were involved in the awareness and education components prior to initiation of the study.

\subsubsection{Economic Risks}

This study had no negative economic impact on the participants. In fact, studies have demonstrated that when a facility has implemented a formal antibiotic stewardship program, the facility and residents benefitted financially with a reduction of their pharmacy monthly expenses (Liam, Stuart, \& Kong, 2015). Secondly, a formal antibiotic stewardship program in place is noted to be the reason for a reduction in the facility's return to hospital numbers (Liam, Stuart \& Kong, 2015), thus reducing penalties imposed by the Centers for Medicare and Medicaid Services. 
Provision eight of the ANA code of ethics demands that nurses collaborate with other health professionals to reduce health disparity (ANA, 2015; Winland-Brown, Lachman, \& Swanson, 2015). When unnecessary expenses are eliminated, the money saved can be reallocated to focus on reducing health disparity in other areas (Winland-Brown, Lachman, \& Swanson, 2015). Patients, families, and healthcare providers must work together to understand the importance of appropriate use of antibiotics within the community to ensure high quality, long-life for the residents and has the potential to decrease economic burden related to unnecessary antibiotic use (Lim, Stuart, \& Kong, 2015).

\subsubsection{Privacy/Data Collection}

Resident data was protected per federal law and facility policy. The researcher was required to review the facility policy related to the current federal laws regarding the Health Insurance and Portability Act of 1996 (HIPAA). The researcher agreed to comply with the facility policy and the federal laws during this study. No protected resident information was included in the reporting of tracking results, chart reviews or evaluation of this project.

\subsubsection{Permission to Utilize AHRQ Guidelines/Forms/Toolkits}

AHRQ (2018) developed an EBP toolkit for long-term care facilities to utilize when implementing an antibiotic stewardship program. The toolkit is free and available via the web site for use by all facilities across the globe. An email sent to the AHRQ permission department was initiated on July 12, 2013. The email was acknowledged on that day and stated that a decision will be made within 7-10 business days. The authorized permission to utilize the AHRQ tools was granted on July 20, 2018(Agency for Health Care Quality, 2018).

\subsubsection{Conflicts of Interest's Evaluation}

There were no conflicts of interest in this study. The researcher was not employed at the clinical site and no compensation was exchanged for the face-to-face educational sessions, attendance at resident council meetings, or participation in the policy and procedure development committee contribution.

\subsubsection{Potential Bias Evaluation}

In the study design, a selection/participation bias was identified. The bias rests in the fact that employees are required to follow policy and that the resident's care is prescribed by the healthcare professionals, who also must follow policy. Therefore, it is the authors' belief that all nursing staff and prescribers will adopt the new policy and participate in the study.

To offset this potential bias, the awareness campaign and the education session was developed to inspire healthcare providers, resident families and residents to acquire current knowledge about appropriate antibiotic use within the long-term care facility. Knowledge brings power to the desired change (Graham, 2006). The knowledge to action theory (KTA) (Graham, 2006) was specifically chosen for this research project to combat this bias. The KTA theory guided change within the facility by empowering the staff with EBP knowledge to want to change their practice.

The second potential bias is found in the data analysis. This researcher firmly believes that this study will demonstrate a change. This bias may naturally occur because of the researcher's belief in the literature that was researched for this project and the researcher is passionate about the desire to make a change in the long-term care environment. Like most researchers, it is natural to want to prove that one's theory is correct (Smith \& Noble, 2014). To minimize this bias, the researcher will consult with an outside statistician to complete data analysis. Translating the knowledge discovered from EBP research into daily clinical practice is a significant and valid contribution to improving overall healthcare outcomes across the globe (Institute of Medicine, 2001; National Strategy for Quality Improvement in Healthcare, 2012).

\section{Results}

\subsection{Study Outcomes}

There was a decrease in the number of antibiotics prescribed for respiratory illness at this facility during September 1, 2018 to November 10, 2018. Ten residents exhibited respiratory illnesses during the early part of flu and influenza season. Three received antibiotic treatment (antibiotic start rate of 0.81) for seven days, thus the total antibiotic days of treatment (DOT) for this time frame was 21 days. The 10-week rate of DOT for 2018 is calculated to be 5.68. The suspected LRI-SBAR form was initiated in all ten respiratory illnesses in the facility and assisted in determining the need for antibiotic use versus management of cold symptoms through other types of medication (decongestants, cough medications).

The 2018 data was compared to the previous year's data (September 1 - November 10, 2017) at this facility. The 2017 data revealed that twelve residents exhibited symptoms of respiratory illness during the early part of flu and 
influenza season. In 2017, no SBAR forms were utilized to communicate clinical symptoms to the prescriber. Eleven of the residents received a 14-day treatment of antibiotics (antibiotic rate of antibiotic starts of 3.37), resulting in a total antibiotic DOT of 154 for this facility during 2017. The 10-week rate of DOT for 2017 was calculated to be 47.15 . This data demonstrates an $87 \%$ decrease in the number of antibiotic DOT for respiratory illness within this facility.

The total number of antibiotics initiated for respiratory illnesses in this facility was 11 in 2017 . Post-intervention of the evidence-based suspected LRI-SBAR tool, the total number of antibiotics starts for respiratory illness in the ten-week tracking period of 2018 was reduced to three. Both years found that no resident returned to the hospital due to the antibiotic being prescribed or not prescribed. When comparing overall categories of "antibiotics given for respiratory illness within the facility" to "no antibiotics given for respiratory illness" a chi-square statistic of 8.9637 with a $p$-value of .002754 further supports the results of this study to be statistically significant when $p<0.5$ (see Table 1 ).

Table 1. Chi-Square calculations

\begin{tabular}{lllc}
\hline Year & Received Antibiotics & No Antibiotics & Statistics \\
\hline $\mathbf{2 0 1 7}$ & 11 & 1 & Row totals \\
\hline Expected Cell Totals & 7.64 & 4.36 & 12 \\
\hline Chi-Square Statistics & 1.48 & 2.59 & 10 \\
\hline $\mathbf{2 0 1 8}$ & 3 & 7 & \\
\hline Expected Cell Totals & 6.36 & 3.64 & \\
\hline Chi-Square Statistics & 1.78 & 8 & $\mathrm{P}<0.5$ \\
\hline Column Totals & 14 & p-value .002754 & \\
\hline
\end{tabular}

Source: Data collection, 2018

Additionally, the overall point-prevalence rate of residents that were on antibiotics during the 10 -week tracking time frames decreased from $25 \%$ in 2017 to just 6\% in 2018 (see Table 2). The overall outcomes for this study were achieved. The results of this pilot study demonstrated a decrease in the number of unnecessary antibiotics for respiratory illness in a long-term care setting. The results strongly support an intentional, multidisciplinary creation and implementation of an antibiotic stewardship program in a long-term care facility can decrease the number of unnecessary antibiotic prescriptions for the residents and ultimately improve resident healthcare outcomes. The results also support that this study design could be easily replicated in other long-term care facilities to improve overall outcomes throughout the long-term care industry.

Table 2. Residents of antibiotic therapy during a 10-week period

Tracking Measurements $\quad 9 / 1 / 17-11 / 10 / 17 \quad 9 / 1 / 2018-11 / 10 / 17 \quad$ Change

September 1 - November 10

\begin{tabular}{lccc}
\hline \multicolumn{3}{c}{ Antibiotic Data } & \\
\hline Antibiotic Days of Treatment (DOT) & 11 & 3 & -7 \\
\hline 10- week Rate of DOT & 47.15 & 5.68 & -41.5 \\
\hline 10-week Rate of antibiotic starts & 3.37 & 0.81 & -2.56 \\
\hline 10-week Point-Prevalence Rate & $24 \%$ & $6 \%$ & -18 \\
\hline Total number of DOT during 10-week & 154 & 21 & $+28 \%$ \\
\hline$\%$ of antibiotic orders complete & Ancillary Data & $100 \%$ & -133 \\
\hline
\end{tabular}




\begin{tabular}{lccc}
\hline$\%$ of suspected LRI followed guidelines & $18 \%$ & $100 \%$ & $+82 \%$ \\
\hline$\%$ of LRI utilized SBAR form & $0 \%$ & $100 \%$ & $+100 \%$ \\
\hline Total \# of resident/family complaints & 0 & 1 & +1 \\
\hline Total \# of return to hospitals & 0 & 0 & 0 \\
\hline Cost of Antibiotics & $\$ 389.26$ & $\$ 65.47$ & $-\$ 323.79$ \\
\hline \# of residents that developed C-Diff & 3 & 0 & -3 \\
\hline \# of adverse reactions to antibiotic & 0 & 0 & 0 \\
\hline
\end{tabular}

\subsection{Observed Associations}

The successful outcomes of this project relied heavily on the support and buy-in from all of the stakeholders. The facility administration was highly motivated to improve their current antibiotic stewardship efforts. This strong dedication of the administrators, residents, and staff was observed to be an unexpected, yet positive, impact on the results of this study. The collaboration that occurred with this researcher and the facility was a significant contribution to the impressive results of this study.

The researcher is aware that many facilities will struggle with the implementation of a similar study to improve outcomes since long-term care administration changes frequently. During this study, the facility did experience a change in the director of nursing in August of 2018. With the help and support of the administrator, the new director of nursing easily transitioned and was supportive of the study and was invested in making a change for the residents of the facility.

A second observation about the results of this study is to address the fact that the early part of cold and influenza season of 2017 may not be equal in severity when compared to the early part of cold and influenza season of 2018. The results could be impacted because perhaps the 2017 flu season was more severe than in 2018 . Although the Indiana State influenza weekly tracking further validates the influenza season of 2017 to be comparable to the influenza season of 2018.

According to the Indiana State Department of Health website (2018) the total number of influenza-like illnesses (ILI) in Indiana at the end of the week of November 11, 2017, was 64. The ILI in Indiana at the end of the week of November 10, 2018, was 76. This data can be broken down to report the number of ILI cases for Indiana residents over the age of 65 . In 2017, two residents over the age of 65 were identified as having ILI, compared to five documented cases of ILI for residents over the age of 65 in 2018.

This data supports that the early start of cold and influenza seasons has been similar, yet for residents over the age of 65, 2018 is showing an increase in the number of ILI cases across Indiana. The results of the pilot study found that more antibiotics were prescribed in 2017 than in 2018. If the documented cases of ILI in Indiana has increased in 2018, one would expect that the number of antibiotics would be increased during 2018.

This study demonstrates that the intervention of utilization of the suspected lower respiratory SBAR has made an impact in determining which resident would benefit from antibiotic treatment. In 2017, without the use of the suspected LRI-SBAR form, it appears that many residents did not benefit from the course of antibiotic treatment. In fact, three residents developed C-Diff infections in 2017 ten-week data collection time frame compared to no residents in the 2018 ten-week data collection time frame.

\subsection{Unintended Consequences}

During the ten-week data collection period, it was determined that a resident did not meet criteria for an antibiotic to be started. Decongestant and cough medications were prescribed for this resident. The family of the resident was adamant about her mother taking an antibiotic for her illness.

To address this concern, the facility nursing staff, director of nursing, medical director, and even housekeeping staff was able to articulate the reason that an antibiotic would not benefit this resident. The direct care nurse first started explaining why the antibiotic was not required based on her symptoms, later in the day the director of nursing confirmed the newly adopted criteria for starting antibiotics with the family member. The director of nursing arranged for the medical director to talk with the distraught family member on the day of the concern and assured her that this is the best plan of action for her mother. Finally, the housekeeper, throughout the normal course of her day, shared that the facility had learned a lot about what happens when a person takes an unnecessary antibiotic. 
The unintended consequence was the ability of the facility to act in concert to make a change. Resistance is the expectation when change is initiated. However, in the development stages of this project, everyone was involved and engaged in the idea that antibiotics could lose their effectiveness if nothing changed in the way the facility utilized antibiotics. The engagement of the entire facility was desired by this researcher, but how cohesive the facility actually rallied around the antibiotic stewardship program to make changes to the way they practiced within the facility, was a consequence that pushed the results to an impressive change within the facility. The engagement of the facility was a significant factor in improving healthcare outcomes for the residents within the facility.

A second unintended consequence of this project is a change within the understanding of the consulting pharmacist and the role of infection control monitoring within the facility. Currently, the pharmacy tracks the numbers of antibiotics used within each facility, reports monthly to the facility about costs of antibiotic, type of infections found at the facility, and the types of the antibiotics prescribed. Before this project, that is all that the consulting pharmacist did with the information.

At the end of the 10-week data collection period, the consulting pharmacist discussed that her understanding of antibiotic stewardship was changed. Antibiotic stewardship is more than just a report that she sends monthly to the facility. She stated that there is value in an infection control nurse in each facility to focus on true reduction in the number of antibiotics prescribed within the facility. Furthermore, she articulated a desire to engage in intentional conversations with the facility about how to improve practice.

\subsection{Missing Data}

All data was able to be retrieved from paper chart audits, pharmacy reports, infection control logs, return to hospital reports and electronic medication administration records. There was no missing data to report for this study.

\subsection{Key Facilitators/Barriers to Meeting Outcomes}

The success of this project lies in the support and engagement of the stakeholders. In the long-term care industry, the facility administrator sets the expectations and standards. In the partnering facility, the administrator and the director of nursing were highly motivated to demonstrate improvement in the quality of care within their facility. This attitude and engagement of the administration team drove the outcomes for this study.

One barrier was the change of the director of nurses (DON). There have been three different DONs throughout the eleven-month collaboration with this facility. The first DON was passionate to develop a program that would meet the new regulatory requirements for antibiotic stewardship in long-term care. Working with this DON set the stage for the project and helped the researcher build relationships with the administrator, pharmacist, medical director, and president of the resident council.

The second DON was only present for two months. She was willing to let the researcher take the lead on the development of policy for the antibiotic stewardship within the facility and to develop and present education throughout the facility. This DON was instrumental in the acceptance of the charter antibiotic stewardship program and policy.

It was during this phase of development that another barrier appeared. The medical director was concerned that this policy would be telling her what to order and she was resistant to the change. The researcher, administrator, and the DON were able to demonstrate that the suspected LRI-SBAR form included national guidelines and were the current best practice for antibiotic stewardship. Once she was able to further research the identified tool for this study, the medical director was willing to participate in the change and even support the education of the nursing staff.

The third DON was present during the final stages of implementation and the 10-week data collection process. She was instrumental in daily tracking of antibiotic use and suspected LRI-SBAR usage. The third DON also provided ongoing education to the nursing staff and families during the data collection period.

\subsection{Sustainability}

The researcher has intentionally prepared the facility to continue the antibiotic stewardship program upon completion of this DNP project. The newly created policy remains the current guide for antibiotic stewardship in this facility. Standard forms for tracking have been established and will continue after the project ends. The annual state survey process will also help sustain this program as it is a requirement for each facility to have antibiotic stewardship in place. This program will avoid potential citations and fines when the antibiotic stewardship program remains an active part of the facility's everyday practices. 
Antibiotic stewardship training has been added to the annual education calendar for the entire facility in the month of September. Finally, antibiotic stewardship training has been included in the nurse orientation program for all newly hired nurses.

\subsection{Future Practice}

This project has the potential to change practice in the long-term care industry. The design of this project could be easily adapted to decrease antibiotic use in other common infections that occur in the long-term care setting. Utilization of other AHRQ suspected illness SBARs could be integrated into practice and further decrease the overall use of inappropriate antibiotics within the long-term care setting.

This project strongly suggests that the development of multidisciplinary teams within the long-term care setting is of vital importance to advance quality improvement projects within the long-term care environment. Valuing the expertise of other disciplines is vital in bringing changes to the current way long-term care provides healthcare in today's industry. Making sustainable changes in the long-term care facility will require buy-in and participation in multidisciplinary teams.

Recommendations for the future inquiry would include the continuation of tracking results throughout the entire 2018 -2019 influenza season and compare the entire season with the 2017-2018 influenza season. This would allow for a larger sample to give greater documentation to support the results of this project. A continuation of monitoring the results would also help with the sustainability of the newly developed antibiotic stewardship program.

A second suggestion for the inquiry would be to compare two different facilities during the same influenza season. One facility would utilize the suspected lower respiratory SBAR form and the other facility would utilize the standard SBAR form. This would allow for paired-T test analysis and other statistical measures to validate the results of this project.

Finally, future inquiry of other SBAR specific forms is needed to further decrease overall facility antibiotic use. In addition to the LRI-SBAR tool, AHRQ (2018) has developed three additional SBAR specific forms for common infections in the long-term care setting. SBAR forms for urinary tract infections (UTI), skin and soft tissue infections, and gastrointestinal infections are currently available for all long-term care facilities to utilize in decreasing overall antibiotic use within the facility.

\section{Discussion}

\subsection{Summary}

This project demonstrated an $87 \%$ reduction in the number of antibiotics prescribed in a long-term care facility for residents who exhibited respiratory symptoms. In 2017, The KTA theory proved to be effective to guide for this project. The successful reduction of unnecessary antibiotic use in the long-term care setting began with the development of a diverse multidisciplinary team that researched the current best-practice guidelines for residents with a suspected lower respiratory illness.

Using the evidence-based guidelines, the team disseminated the information to the entire facility, including the residents and resident's family to ensure that the new antibiotic stewardship program was embraced by the facility. Continued interaction with the facility and attention to areas of resistance contributed to the successful resident healthcare outcomes.

The strength of this project was that a clinically significant change was made in this long-term care facility. The positive outcomes not only included the reduction in the number of antibiotics prescribed at the facility but the teamwork, among all departments, residents, resident's families, prescribers, facility administration and outside consulting agencies proved to be an effective model to promote a change within the long-term care setting. This collaboration among all departments is a model for making permanent changes in a very challenging setting and could be the foundation in creating change in the long-term care environment.

\subsection{Interpretation}

The interventions, for this project, focused on making changes to the way the nurse, facility staff, facility administration, prescriber, resident and resident's family members interacted when the resident exhibited signs and symptoms of respiratory illness. Careful analysis of the results of this study lead to three themes as essential elements to make changes in the current antibiotic prescribing practice in the long-term care industry.

\subsubsection{Multidisciplinary Team Development}

The long-term care environment is a very social setting. After all, the very first thing that is covered in new 
employee orientation is a conversation about resident's rights and it is made clear that the new employee will be working "in the resident's home." This extreme social focus, within a formal healthcare setting, is unique to most healthcare settings. The long-term care environment is unlike a hospital setting, and all staff members, including dietary and housekeeping heavily impact the daily interactions of the resident while they live at the facility (Fleming et al., 2015; Kistler et al., 2017).

For this reason, it is imperative that long-term care administrators understand that an essential part of making a change within the long-term care environment is an intentional effort to be all-inclusive with every department when change is desired. Fleming et.al, 2015 supports this idea of community as essential in transforming current practices in long-term care settings.

In this study, one key component was to include multiple different disciplines at the development stages of the antibiotic stewardship program. Nursing, pharmacy, medical prescribers lead the development in the antibiotic program, but it was imperative to hear from resident's, housekeeping, certified nursing assistance, dietary, and resident's family. Graham's (2006) KTA model guided this inclusive idea throughout the entire project development phase, implementation phase, and monitoring phase. Valuing all employees that work in the long-term care environment contributed to the effective change that the results demonstrated in this practice change. Understanding the importance of the long-term care social environment will be essential in sustaining the change at this facility.

\subsubsection{Comprehensive Education}

Knowledge sharing is always an important part of a change in practice (Fleming et al., 2015; Kistler, 2017). The KTA model identifies knowledge as the foundation to change (Graham et al., 2006). This endeavor supports the idea that the practice change in this study benefitted from including all members of the facility, including residents' and their family members in the awareness campaign before the change in practice took place.

Long-term care environments need to remember that they need to include the resident and their family members in the change (Kistler, 2017). This strategy is frequently forgotten in this setting, as the drive for change is regularly focused on the direct nursing care staff (Fleming et al., 2015).

\subsubsection{Easily Integration of an Evidence-Based Tool}

The long-term care environment is a complex system that is driven by a long list of regulations. Fleming et al., (2015) noted that successful change in practice will only occur if the intervention is not overwhelming to the staff. This project benefitted from making a slight, but significant change in one document. The facility had previously adapted to utilization of the standard SBAR form in their daily practice. This push in long-term care for a standard communication tool between the nurse and other medical providers started in 2010 (Kistler, 2017). The suspected LRI SBAR form was only slightly different than what the nurses had been documenting on for years.

By utilizing a familiar form, the nurses were less resistant to the change (Kistler, 2017). The integration of the practice algorithm guidelines, found on the second page of the suspected LRI SBAR form, was new to the nursing staff but the form was a familiar part of their daily practice. Therefore, the transition to utilizing a specific focused SBAR form did not appear to cause distress among the nursing staff during this project implementation to change current prescribing practices.

Each long-term care facility has a unique vibe to the way it provides care to the residents. Understanding that a tailored intervention will be an important consideration when tackling an antibiotic stewardship program in each facility to ensure the antibiotic stewardship program remains effective and sustainable with a unique environment.

\subsubsection{Financial Savings}

The financial savings to this facility as a result of the development of an antibiotic stewardship program change was found to be $\$ 323.79$ savings over 10 weeks. The potential yearly saving could be close to $\$ 1000 /$ year if the antibiotic stewardship programs are sustainable at this facility.

\section{Project Limitations}

This project was completed in one small facility in Indiana with a small number of nursing staff (total of 12). The study can be replicated in larger facilities but will require a greater time commitment to educate the entire facility staff, residents, resident families, and administration team.

Resistance to the new antibiotic stewardship may be greater with larger facilities and larger resident populations. Therefore, the design of the study may need to be altered in larger facilities to ensure that one specific nurse is 
assigned to implement and monitor the program to ensure that the antibiotic rates decrease.

It is important to recognize that the results of this study may have been impacted by the design method. The researcher was highly engaged in keeping the awareness of the antibiotic stewardship program in the minds of everyone at the facility. The researcher visited the long-term care facility weekly throughout the ten-week data collection timeframe. This presence and intentional on-going conversation with the facility staff, administration, residents and family members may have contributed to the positive results of this study.

The facility does not have a dedicated infection control nurse due to the size of the facility and budgetary limitations. The current infection control monitoring is shared between the unit manager and DON. The researcher stepped in to focus on the change but realizes that the sustainability of this program might fade when the study is complete.

To minimize the effect of the design in the study and sustainability of the program, the researcher intentionally began to make the weekly visits much shorter in duration at weeks $6-10$. This allowed for the facility staff to identify an "antibiotic stewardship program champion" to be the face of the program, instead of the researcher.

\section{Conclusions}

This project has the potential to change the current practice in long-term care. The idea of antibiotic stewardship is not going away any time soon for the long-term care industry. This project provides a useful and practical way to make antibiotic stewardship programs meaningful to the residents and staff in the long-term care setting. It also provides an example of how to effectively decrease the use of unnecessary antibiotics within the long-term care environment without adding budgetary expenses.

Bringing an evidence-based tool to the bedside to improve overall healthcare outcomes for the residents living in a long-term care environment was effectively demonstrated in this project. Integration of best practice at the bedside is an important concept to improve healthcare in everyday practice. This project demonstrated that the use of validated tools can be quickly integrated into practice and produce effective change within as little as ten weeks.

Careful attention was placed on ensuring this project would be sustainable after the project ended. Engagement of the facility staff from day one of this project allowed ownership of the project to remain firmly at the facility. Annual state survey process evaluates the effectiveness of antibiotic stewardship in the facility every year. Therefore, sustainability will be enhanced by the enforcement of regulations and the facility has a great incentive to keep the antibiotic stewardship active and effective.

The study demonstrated the effectiveness of one person overseeing the focus on infection control within the building. Throughout this project, the facility sees the value of making changes within staffing to continue positive resident healthcare outcomes. The facility has begun conversations about the possibility of dedicating one nurse to the role of infection control/education. The awareness of the importance of infection control within the building will assist with the sustainability of this project.

The project design could be the foundation in any long-term care environment for the development of an antibiotic stewardship program. It provides a theoretical basis for developing and sustaining effective change in prescribing practices with the long-term care environment. This project also provides an example of how facilities can easily implement evidence-based tools at the bedside to improve overall healthcare outcomes for the residents.

Future research is needed to explore the effectiveness and sustainability of antibiotic stewardship programs across the globe in long-term care settings. Replication of this study, using other disease-specific SBAR tools, are needed to substantiate the results of this study. Attempting this study in larger long-term care facilities will also provide valuable information about decreasing the use of unnecessary antibiotics in the long-term care setting.

Next steps include dissemination of what was learned through this project. Sharing the information and knowledge gained will be essential in making improvements in decreasing the number of inappropriate antibiotics that are prescribed to residents within the long-term care setting. All residents will benefit from knowledgeable leadership in the long-term care industry.

Timely distribution of recent successful models of antibiotic stewardship can be a powerful technique to help leaders visualize success in their own facilities. An effective antibiotic stewardship program, in the long-term care setting, is not an impossible task. Sharing the results of this project will provide a practical way for the nursing home industry to begin to change the current practice of overuse, misuse, or unnecessary use of 
antibiotics for the long-term care resident.

\section{Acknowledgements}

The author acknowledges sincere gratitude for the following mentors in the development of this project. Patricia A. Keresztes, $\mathrm{PhD}$, MSN for your guidance in implementation of this project and your constant encouragement to keep moving forward when writing became difficult. Corrine Cochran, DNP, NNP-BC for your critical guidance and precision in the development of the PICOT question that grounded this project. Amberly Wagner-Connolly, DNP, NNP-BC for your guidance and encouragement all through the DNP capstone experience.

\section{References}

Agency for Healthcare Research and Quality. (n.d.). Toolkit 1: Start an antibiotic stewardship program. Retrieved form https://www.ahrq.gov/nhguide/toolkits/implement-monitor-sustain-program/toolkit1-start-program.html

Aryee, A. (2015). Antimicrobial stewardship-can we afford to do without it? British Journal of Clinical Pharmacology, 79 (2), 173.

Ashraf, M., \& Cook, P. (2016). Antibiotic misuse in hospital, outpatient, and long-term care settings. North Carolina Medical Journal, 77(5), 346-349.

Center for Medicare and Medicaid Programs. (n.d.). Reform of Requirements for Long-Term Care Facilities. Retrieved July 16, 2015, from http://1.usa.gov/1hIe6Du

Centers for Disease Control and Prevention. (n.d.). Core Elements of Antibiotic Stewardship for Nursing Homes. Retrieved from www.cdc.gov/longtermcare/prevention/antibiotic-stewardship.html

Charani, E., \& Holmes, A. H. (2013). Antimicrobial stewardship programmes: The need for wider engagement. BMJ Quality \& Safety, 22(11), 885.

Cranley, L. A., Norton, P. G., Cummings, G. C., Barnard, D., Brata-Garga, \& Estabrooks, C. A. (2012). Identifying resident care areas for a quality improvement intervention in long-term care: A collaborative approach. BMC Geriatrics, 12(1), 59.

Crnich, C. J., Jump, R., Trautner, B., Sloane, P. D., \& Mody, L. (2015). Optimizing antibiotic stewardship in nursing homes; A narrative review and recommendation for improvement. Drugs \& Aging, 32(9), 699-716.

Daneman, N. M., Campitelli, M. A., Giannakeas, V., Morris, A. M., Bell, C. M, Maxwell, C. J., ... Bronskill, S. E. (2017). Influences on the start, selection and duration of treatment with antibiotics in long-term care facilities. Canadian Medical Association Journal, 189(25), E851-E860.

Dar, O. A., Hasan, R., Schlundt, J., Harbath, S., Cleoa, G., Dar, F. K., ... Heyman, D. L. (2016). Exploring the evidence base for national and regional policy interventions to combat resistance. The Lancet, 387(10015), 285-295.

Del Arco, A., Tortajada, B., De, I. T., Olalla, J., Prada, J. L., Fernandez, F....Montiel, N. (2015). The impact of antimicrobial stewardship programme on the use of antimicrobials and the evolution of drug resistance. European Journal of Clinical Microbiology and Infections Disease, 34(2), 247-251.

Diamond, V. J. (2016). Strength in numbers. Healthcare Purchasing News, 40(11), 20-25.

Doran, S., Nadkarni, L., Lyn Price, L., Lawrence, K. P., Davidson, L. E., Evan, J., ... Snydman, D. R. (2013). A nationwide survey of antimicrobial stewardship practices. Clinical Therapeutics, 35(6), 758-765.

Dubberke, E. R., Olsen, M. A., Stwalley, D., Kelly, C. P., Gerding, D. N., Young-Xu, Y., \& Mahe, C. (2016). Identification of Medicare recipients at highest risk for clostridium difficille infection in the US by population attributable risk analysis. PloS One, 11(2).

Dyar, O. J. (2015). Strategies and challenges of antimicrobial stewardship in long-term care facilities. Clinical Microbiology and Infection, 21(1), 973.

Evans, G. (2013). CDC looking to CMS to add "teeth" to new antibiotic stewardship guidelines. Hospital Infection Control \& Prevention, 40(12).

Fleming, A., Bradley, C., Cullinan, S., \& Byrne, S. (2015). Antibiotic prescribing in long-term care facilities: A meta-synthesis of qualitative research. Drugs \& Aging, 32(4), 295-203.

Gandra, S. (2014). Economic burden of antibiotics resistance: how much do we really know? Clinical 
microbiology and infection, 20(10), 973.

Gifford, J., Vaeth, E., Richards, K., Siddiqui, T., Gill, C., Wilson, L., \& DeLisie, S. (2017). Decision support during electronic prescription to stem antibiotic overuse for acute respiratory infections: A long-term, quasi-experimental study. BMC Infectious Diseases, 17.

Graham, I. D., Logan, J., Harrison, M. B., Straus, S. E., Tetroe, J., Caswell, W., \& Robinson, N. (2006). Lost in knowledge translation: Time for a map? Journal of Continuing Education in the Health Professions, 26(1), 13.

Hertz, B. T. (2015). Germ warfare; a tea, approach can reduce the misuse of antibiotics in log-term care. Long-term, living, 64(6), 16.

Lenhoff, A. (2016). In the news; Antibiotic resistance. Medical Laboratory Observer, 48(1), 20-25.

Lim, C. J., Stuart, R. L., \& Kong, D. C. (2015). Antibiotic use in residential aged care facilities. Australian Family Physicians, 44(4), 192-196.

Neville, S., Napier, S., Adams, J., Wham, C., \& Jackson, D. (2016). An integrative review of factors related to building age-friendly rural community. Journal of Clinical Nursing, 25, 17-18.

Olans, R. D., Nicholas, P. K., Hanely, D., \& DeMaria, A. (2015). Defining a role for nursing education in staff nurse participation in antimicrobial stewardship. The Journal of Continuing Education in Nursing, 46(7), 318-321.

Pogue, J. M. (2015). Appropriate antimicrobial therapy in the era of multidrug-resistant human pathogens. Clinical Microbiology and Infection, 21(4), 302.

Prina, E., Ranzani, O. T., \& Torres, A. (2015). Community-acquired pneumonia. The Lancet, 386(9998), 1097-1108.

Romick-Rosendale, L., Legomarcino, A., Patel, N. B., Morrow, A. L., \& Kennedy, M. A. (2014). Prolonged antibiotic use induced intestinal injury in mice that is repaired after receiving antibiotic pressure: Implications for empiric antibiotic therapy. Metabolomics, 10(1), 8-20.

Sloane, P. D., Huslage, K., Kistler, C. E., \& Zimmerman, S. (2016). Optimizing antibiotic use in nursing homes through antibiotic stewardship. North Carolina Medical Journal, 77(5), 324-329.

Squires, J. E., Sullivan, K., Eccles, M. P., Worswick, J., \& Grimshaw, K., M. (2014). Are multifaceted interventions more effective than single-component interventions in changing health-care professionals' behavior? An overview of systemic reviews. Implementation Science, 9, 152.

Van de Berg, P., Kemperman, A., De Kleijn, B., \& Borgers, A. (2015). Locations that support social activity participation of the aging population. International Journal of Environmental Research and Public Health 12(9), 10432-10449.

Voelker, R. (2015). Nursing homes need improved antibiotic stewardship. Journal of American Medical Association, 314(16), 1685-1685.

\section{Copyrights}

Copyright for this article is retained by the author(s), with first publication rights granted to the journal.

This is an open-access article distributed under the terms and conditions of the Creative Commons Attribution license (http://creativecommons.org/licenses/by/4.0/). 